\title{
WEAK AND SMOOTH SOLUTIONS FOR A FRACTIONAL YAMABE FLOW: THE CASE OF GENERAL COMPACT AND LOCALLY CONFORMALLY FLAT MANIFOLDS
}

\author{
PANAGIOTA DASKALOPOULOS, \\ YANNICK SIRE \\ AND JUAN-LUIS VÁZQUEZ
}

\begin{abstract}
As a counterpart of the classical Yamabe problem, a fractional Yamabe flow has been introduced by Jin and Xiong (2014) on the sphere. Here we pursue its study in the context of general compact smooth manifolds with positive fractional curvature. First, we prove that the flow is locally well posed in the weak sense on any compact manifold. If the manifold is locally conformally flat with positive Yamabe invariant, we also prove that the flow is smooth and converges to a constant scalar curvature metric. We provide different proofs using extension properties introduced by Chang and González (2011) for the conformally covariant fractional order operators.
\end{abstract}

\section{Contents}

1. Introduction 2

1.1. The nonlocal flow 2

1.2. General flow problem and results 3

1.3. Formulation as a fractional fast diffusion 4

2. Conformal fractional Laplacians 5

2.1. Poincaré-Einstein manifolds and Graham-Zworski theory

2.2. Conformal fractional Laplacians 6

2.3. Connection to Dirichlet-to-Neumann operators 7

3. Short time existence for general compact manifolds 8

4. Global existence and proof of Theorem 1.2

Acknowledgments 16

References 16 


\section{INTRODUCTION}

Given a compact Riemannian manifold $\left(M, g_{0}\right)$ of dimension $n \geq 2$, Hamilton introduced in [Ham88] the following evolution for a metric $g(t)$

$$
\left\{\begin{array}{l}
\partial_{t} g(t)=-\left(R_{g(t)}-r_{g(t)}\right) g(t) \\
g(0)=g_{0},
\end{array}\right.
$$

where $R_{g(t)}$ is the scalar curvature of $g(t)$ and

$$
r_{g(t)}=\operatorname{vol}_{g(t)}(M)^{-1} \int_{M} R_{g(t)} d \operatorname{vol}_{g(t)} .
$$

This gave rise to an extensive literature, see e.g. [Cho92, Ye94, SS03, Bre05, Bre07. On the other hand, in a seminal paper GZ03] Graham and Zworski constructed a family of conformally covariant operators $P_{\gamma}^{g}, \gamma \in(0, n / 2)$, on the conformal infinity of a Poincaré-Einstein manifold. These operators appear to be the higher-order generalizations of the conformal Laplacian. They coincide with the GJMS operators of GJMS92 for suitable integer values of $\gamma$. This paved the way to define an interpolated quantity $Q_{\gamma}^{g}$ for each $\gamma \in(0, n / 2)$, which is just the scalar curvature for $\gamma=1$, and the $Q$-curvature for $\gamma=2$ (see Section 2.2). This new notion of curvature has been investigated in GQ13, CG11, GMS12.

1.1. The nonlocal flow. The Graham-Zworski approach motivates the introduction of a new flow problem with fractional curvature that replaces the curvature in Hamilton's Yamabe flow Ham88] by the new curvatures. The problem is posed as follows: Given a compact Riemannian manifold $\left(M^{n}, g_{0}\right)$ of dimension $n \geq 2$ and given $\gamma \in(0, n / 2)$, to find an evolving metric $g(t)$ on $M$ such that

$$
\left\{\begin{array}{l}
\partial_{t} g=-k(t)\left(Q_{\gamma}^{g(t)}-q_{\gamma}^{g(t)}\right) g(t) \\
g(0)=g_{0}
\end{array}\right.
$$

where

and

$$
k(t)=\frac{n-2 \gamma}{2 n} \operatorname{vol}_{g(t)}(M)^{\frac{2 \gamma-n}{n}}
$$

$$
q_{\gamma}^{g}=\operatorname{vol}_{g(t)}(M)^{-1} \int_{M} Q_{\gamma}^{g} d \operatorname{vol}_{g}
$$

Note that $k(t)$ and $q_{\gamma}^{g}$ depend only on $t$, and denote (see [CG11])

$$
Q_{\gamma}^{g}=P_{\gamma}^{g}(1)
$$

This flow is the gradient flow of the normalized total $\gamma$-curvature functional

$$
\mathcal{S}_{\gamma}(g)=\operatorname{vol}_{g}(M)^{\frac{2 \gamma-n}{n}} \int_{M} Q_{\gamma}^{g} d \operatorname{vol}_{g}
$$

where $g \in\left[g_{0}\right]$, the conformal class of $g_{0}$, as observed in [JX14]. For $\gamma=1$, the defined flow is just the Yamabe flow introduced by Hamilton. This new geometrical problem has been already considered by Jin and Xiong in [JX14] where the authors 
investigate the flow on the sphere $M=\mathbb{S}^{n}$ with the round metric. They introduce the flow actually in this context but the generalization on any compact manifold $M$ is straightforward. An important property of the previous flow is that it conserves the volume in time.

1.2. General flow problem and results. The aim of the present paper is twofold. We first prove existence and uniqueness of mild and weak solutions of the fractional flow on any compact manifold with positive fractional curvature and then move on to the case of locally conformally flat manifolds with non-negative Yamabe invariant in the spirit of Ye's work [Ye94]. For reasons which will become transparent later, we study the flow (1.2) for $\gamma \in(0,1)$, and this assumption is kept throughout the paper.

We prove the following results.

Theorem 1.1. Assume that $g(0) \in\left[g_{0}\right]$ is smooth and that $M$ is an n-dimensional smooth compact boundaryless manifold, being the conformal infinity of a PoincaréEinstein manifold $\left(X^{n+1}, g_{+}\right)$. Assume also that $Q_{\gamma}^{g_{0}} \geq 0$ and $\lambda_{1}\left(g_{+}\right) \geq \frac{n^{2}}{2}-\gamma^{2}$. Then the flow in (1.2) with initial metric $g(0)$ exists for all times in the sense of mild solutions and weak solutions if $\gamma \in(0,1 / 2]$, and provided $H=0$ in the case $\gamma \in(1 / 2,1)$. Here $H$ denotes the mean curvature of $\partial_{\infty} X=M$.

A number of remarks follow:

(i) A reminder of the concepts of the Graham-Zworski theory is given in Section 2 ,

(ii) In the previous theorem, by mild sense, we mean that the flow exists for all times using a semi-group approach. It basically means that, as soon as a contraction property is satisfied, the Crandall-Liggett theorem [CL71] ensures the existence of a mild solution as limit of the Implicit Time Discretization Scheme. Then we connect to weak solutions of the flow.

(iii) The present paper deals with flows with non-negative fractional curvarture. Due to our approach, we are not able to deal with negative $Q_{\gamma}^{g_{0}}$ curvature. This is due to the fact that in this case one cannot show contractivity of the semi-group approach in the Crandall-Liggett theory. We will leave it as an open problem and hope to investigate it in the next future. However, a remark needs to be done in the negative case. Indeed, if one considers a constant $Q_{\gamma}^{g_{0}}$ curvature equal to -1 and space-independent solutions, one gets the following ODE for the un-rescaled flow on $M$ (see (3.2))

$$
\partial_{t} U^{N_{\gamma}}=-U, \quad N_{\gamma}=\frac{n+2 \gamma}{n-2 \gamma}
$$

Since $N_{\gamma}>1$, one has two different solutions, one being trivial $U(t) \equiv 0$ and the other one non trivial $U(t)=k t^{1 /\left(N_{\gamma}-1\right)}$. This is a counterexample to uniqueness. 
In order to state our next theorem, we introduce the well-known Yamabe constant: if $(M, g)$ is a compact manifold, then the Yamabe constant $Y(M)$ is the quantity

$$
Y(M):=\inf \left\{\frac{\int_{M} R_{h} d V_{h}}{\left(\int_{M} d V_{h}\right)^{\frac{n-2}{n}}}, h \in[g]\right\}
$$

where $R_{h}$ is the scalar curvature of $M$ with respect to the metric $h$.

Theorem 1.2. Let $\gamma \in(0,1)$ and assume that $g(0) \in\left[g_{0}\right]$ is smooth, has nonnegative fractional curvature, and that $M$ is locally conformally flat with positive Yamabe constant $Y(M)$. Then the flow in (1.2) with initial metric $g(0)$ exists for all times $t \in(0,+\infty)$ and is smooth. Furthermore, there exists a smooth metric $g_{\infty}$ such that

$$
\lim _{t \rightarrow+\infty}\left\|g(t)-g_{\infty}\right\|_{C^{\ell}}=0
$$

for any integer $\ell$, and $Q_{\gamma}^{g_{\infty}}$ is constant.

1.3. Formulation as a fractional fast diffusion. A main feature of the method developed in the present paper is to provide a solution to the fractional Yamabe problem by parabolic arguments. An important property of the operators $P_{\gamma}^{g}$ is their conformal covariance. More precisely, as usual we write $g=u^{4 /(n-2 \gamma)} \bar{g}$, then we have for any $f \in C^{\infty}(M)$ the conformal law (see [GZ03])

$$
P_{\gamma}^{\bar{g}}(u f)=u^{\frac{n+2 \gamma}{n-2 \gamma}} P_{\gamma}^{g}(f)
$$

Indeed, the metric $g_{\infty}$ in Theorem 1.2 satisfies

$$
P_{\gamma}^{g_{\infty}} u_{\infty}=Q_{\gamma}^{g_{\infty}} u_{\infty}^{\frac{n+2 \gamma}{n-2 \gamma}} \text { on } M
$$

where $Q_{\gamma}^{g_{\infty}}$ is constant and $g_{\infty}=u_{\infty}^{\frac{4}{n-2 \gamma}} g_{0}$. The fractional Yamabe problem has been investigated for $\gamma \in(0,1)$ in GQ13 and in the case of locally conformally flat manifolds in QR06 with positive Yamabe constant (with an additional assumption on the Poincaré exponent of the Kleinian group). See also [KMW]. In the case of the sphere (or $\mathbb{R}^{n}$ ), the classification of solutions of the Yamabe equation has been obtained in [CLO06, Li04].

As already noticed in the case of the standard Yamabe flow, the fractional Yamabe flow (1.2) is related to a porous medium type equation. Indeed, one can eliminate the factor $k(t)$ by a time rescaling, so that our nonlocal flow changes into the following Cauchy problem

$$
\left\{\begin{array}{l}
\partial_{t} g=\left(q_{\gamma}^{g}-Q_{\gamma}^{g}\right) g \\
g(0)=g_{0}
\end{array}\right.
$$


where $t$ denotes now the new time. Furthermore, if we write $g(t)=u^{\frac{4}{n-2 \gamma}} g_{0}$, this Cauchy problem reduces to

$$
\left\{\begin{array}{l}
\partial_{t}\left(u^{N_{\gamma}}\right)=-P_{\gamma}^{g_{0}} u+q_{\gamma}^{g(t)} u^{N_{\gamma}}, x \in M \\
u(0)=u_{0},
\end{array}\right.
$$

where $N_{\gamma}=\frac{n+2 \gamma}{n-2 \gamma}$, up to a numerical constant that is absorbed into the time variable. Since $N_{\gamma}>1$, this is a fast diffusion equation of fractional type on the manifold $M$, a very convenient formulation for our calculations.

- We recall that when $M=\mathbb{R}^{n}$ with the flat metric (or when $M$ is the sphere through stereographic projection), Equation (1.7) becomes

$$
\left\{\begin{array}{l}
\partial_{t} u^{N_{\gamma}}=-(-\Delta)^{\gamma} u, \quad x \in \mathbb{R}^{n}, \\
u(0)=u_{0},
\end{array}\right.
$$

where $(-\Delta)^{\gamma}$ is the so-called fractional Laplacian, i.e., the Fourier multiplier with symbol $|\xi|^{2 \gamma}$. Such an equation has been investigated in dPQRV11 and dPQRV12 and we will make use of several of their techniques in the present paper. Actually, in the paper dPQRV12 the so-called non-rescaled flow is thoroughly investigated.

Remark 1.1. It is important to notice that the case $\gamma=1 / 2$ corresponds actually to Escobar's problem [Esc92]. This has been emphasized in [CG11. This provides a new approach to the scalar curvature flow on manifolds with boundary. We refer the reader to Brendle's [Bre02] for the study of the Yamabe flow on manifolds with boundary.

\section{Conformal fractional Laplacians}

2.1. Poincaré-Einstein manifolds and Graham-Zworski theory. Before proceeding further, we give a summary of the Graham-Zworski theory. Let $M$ be a compact manifold of dimension $n$ with a metric $\hat{g}$. Let $X^{n+1}$ be a compact manifold of dimension $n+1$ with boundary $M$. A function $\rho$ is a defining function of $\partial X$ in $X$ if

$$
\rho>0 \text { in } X, \quad \rho=0 \text { on } \partial X, \quad d \rho \neq 0 \text { on } \partial X .
$$

We say that $g^{+}$is a conformally compact metric on $X$ with conformal infinity $(M,[\hat{g}])$ if there exists a defining function $\rho$ such that the manifold $(\bar{X}, \bar{g})$ is compact for the metric $\bar{g}=\rho^{2} g^{+}$, and $\left.\bar{g}\right|_{M} \in[\hat{g}]$, the conformal class of $\hat{g}$. If, in addition $\left(X^{n+1}, g^{+}\right)$ is a conformally compact manifold and $\operatorname{Ric}\left[g^{+}\right]=-n g^{+}$, then we call $\left(X^{n+1}, g^{+}\right)$a conformally compact Einstein manifold. In the typical example $X$ is the Poincaré disk with the hyperbolic metric and $M$ is the infinite horizon at $|x|=1$.

It is well known that, given a conformally compact, asymptotically hyperbolic manifold $\left(X^{n+1}, g^{+}\right)$and a representative $\hat{g}$ in $[\hat{g}]$ of the conformal infinity $M$, there is a defining function $\rho$ such that, on $M \times(0, \epsilon)$ in $X, g^{+}$has the normal form $g^{+}=\rho^{-2}\left(d \rho^{2}+g_{\rho}\right)$ where $g_{\rho}$ is a one-parameter family of metrics on $M$ such that 
$\left.g_{\rho}\right|_{M}=\hat{g}$. Moreover, $g_{\rho}$ has an asymptotic expansion which contains only even powers of $\rho$, at least up to degree $n$.

Graham-Zworski GZ03] have shown that, given $f \in C^{\infty}(M)$ and $s \in \mathbb{C}$, the eigenvalue problem

$$
-\Delta_{g} u-s(n-s) u=0, \quad \text { in } X
$$

has a solution of the form

$$
u=F \rho^{n-s}+H \rho^{s},\left.\quad F\right|_{\rho=0}=f
$$

for all $s \in \mathbb{C}$ unless $s$ belongs to the spectrum of $\Delta_{g^{+}}$. Moreover, it is known that

$$
\sigma\left(\Delta_{g^{+}}\right)=\left[(n / 2)^{2}, \infty\right) \cup \sigma_{p p}\left(\Delta_{g^{+}}\right),
$$

where the pure point spectrum $\sigma_{p p}\left(\Delta_{g^{+}}\right)$(the set of $L^{2}$ eigenvalues) is finite and it is contained in $\left(0,(n / 2)^{2}\right)$. Now, the scattering operator on $M$ is defined as

$$
S(s) f=\left.H\right|_{M} \text {. }
$$

It is a meromorphic family of pseudo-differential operators in the half-plane $\operatorname{Re}(s)>$ $n / 2$. The values $s=n / 2, n / 2-1, n / 2-2, \ldots$ are simple poles of finite rank, these are known as the trivial poles; $S(s)$ has infinitely many other poles. However, for the rest of the paper we assume that we are not in those exceptional cases.

2.2. Conformal fractional Laplacians. Using the previous notations we define the conformally covariant fractional powers of the Laplacian as follows: for $s=\frac{n}{2}+\gamma$, $\gamma \in\left(0, \frac{n}{2}\right), \gamma \notin \mathbb{Z}$, we set

$$
P_{\gamma}^{\hat{g}}:=d_{\gamma} S\left(\frac{n}{2}+\gamma\right), \quad d_{\gamma}=2^{2 \gamma} \frac{\Gamma(\gamma)}{\Gamma(-\gamma)}<0 .
$$

The previous formula is a straightforward extension of [GZ03] (see also CG11]). In our framework, the idea is to see the compact (smooth connected) manifold $M$ as the boundary infinity of the asymptotical hyperbolic manifold $X^{n+1}$. On the other, with this choice of multiplicative factor, the principal symbol of $P_{\gamma}^{\hat{g}}$ is exactly the principal symbol of the fractional Laplacian $\left(-\Delta_{\hat{g}}\right)^{\gamma}$, precisely,

$$
\sigma\left(P_{\gamma}^{\hat{g}}\right)=|\xi|^{2 \gamma} \text {. }
$$

We thus have that $P_{\gamma}^{\hat{g}}=\left(-\Delta_{\hat{g}}\right)^{\gamma}+\Psi_{\gamma-1}$, where we denote by $\Psi_{m}$ a pseudo-differential operator of order $m$. In the previous formula, the operator $\left(-\Delta_{\hat{g}}\right)^{\gamma}$ is the fractional power of the Laplace-Beltrami operator $-\Delta_{\hat{g}}$ with respect to the metric $\hat{g}$.

When $\gamma$ is an integer, it turns out that the $P_{k}$ are the conformally invariant powers of the Laplacian constructed by Graham-Jenne-Mason-Sparling GJMS92] and Fefferman-Graham [FG02, that are local operators. In particular, when $k=1$ we have the conformal Laplacian,

$$
P_{1}=-\Delta_{\hat{g}}+\frac{n-2}{4(n-1)} R_{\hat{g}}
$$


and when $k=2$, the Paneitz operator

$$
P_{2}=\left(-\Delta_{\hat{g}}\right)^{2}+\delta\left(a_{n} R g+b_{n} R i c\right) d+\frac{n-4}{2} Q^{n} .
$$

The operators $P_{\gamma}^{\hat{g}}$ satisfy an important conformal covariance property (see [GZ03]). Indeed, for a conformal change of metric

$$
\hat{g}_{w}=w^{\frac{4}{n-2 \gamma}} \hat{g},
$$

we have that

$$
P_{\gamma}^{\hat{g}_{w}}=w^{-\frac{n+2 \gamma}{n-2 \gamma}} P_{\gamma}^{\hat{g}}(u \cdot)
$$

Finally, the $Q_{\gamma}$-curvature of the metric associated to the functional $P_{\gamma}$ is defined by

$$
Q_{\gamma}^{\hat{g}}:=P_{\gamma}^{\hat{g}}(1)
$$

In particular, for a change of metric as (2.4), we obtain the equation for the $Q_{\gamma}$ curvature:

$$
P_{\gamma}^{\hat{g}}(w)=w^{\frac{n+2 \gamma}{n-2 \gamma}} Q_{\gamma}^{\hat{g}_{w}}
$$

2.3. Connection to Dirichlet-to-Neumann operators. Let us now describe the Chang-González extension property (see [CG11]). See also [CS07] in the flat case. This is what we really use in the present paper to investigate our flow. The paper establishes a link between the just mentioned family of conformally covariant operators and Dirichlet-to-Neumann boundary operators corresponding to some uniformly degenerate elliptic operators. To be more precise: given an asymptotically hyperbolic manifold $\left(X^{n+1}, g^{+}\right)$and a representative $\hat{h}$ of the conformal infinity $\left(M^{n},[\hat{h}]\right)$, one can find a geodesic defining function $\rho$ such that the compactified metric can be written as

$$
\bar{g}=\rho^{2} g^{+}=d \rho^{2}+h_{\rho} .
$$

Consider now the following boundary value problem

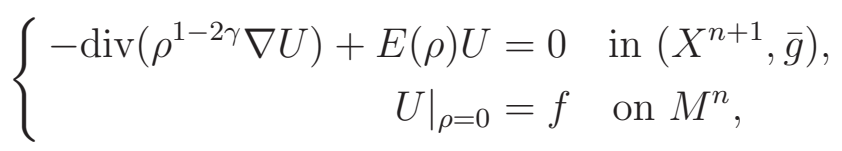

where

$$
E(\rho)=\rho^{-1-s}\left(\Delta_{g^{+}}-s(n-s)\right) \rho^{n-s},
$$

with $s=n / 2+\gamma$. The following has been proved in [CG11], Theorem 5.1.

Lemma 2.1. Let $(\bar{X}, \bar{g})$ be a smooth $(n+1)$-dimensional compact manifold with boundary and let $\hat{g}$ be the restriction of the metric $\bar{g}$ to the boundary $M=\partial_{\infty} X$. Let $\rho$ be a geodesic defining function. Then there exists an asymptotically hyperbolic metric $g^{+}$with $\lambda_{1}\left(g_{+}\right) \geq \frac{n^{2}}{2}-\gamma^{2}$ on $X$ such that when $U$ solves (2.7), then 
(1) For $\gamma \in(0,1 / 2)$,

$$
P_{\gamma}^{\hat{g}} f=-d_{\gamma}^{*} \lim _{\rho \rightarrow 0} \rho^{1-2 \gamma} \partial_{\rho} U
$$

where the value of the constant $d_{\gamma}^{*}$ is given by

$$
d_{\gamma}^{*}:=-\frac{2^{2 \gamma-1} \Gamma(\gamma)}{\gamma \Gamma(-\gamma)}>0 .
$$

(2) For $\gamma=1 / 2$

$$
P_{\frac{1}{2}}^{\hat{g}} f=-\lim _{\rho \rightarrow 0} \partial_{\rho} U+\frac{n-1}{2} H f,
$$

where $H$ is the mean curvature of $M$.

(3) For $\gamma \in(1 / 2,1)$, if $H=0$ we have

$$
P_{\gamma}^{\hat{g}} f=-d_{\gamma}^{*} \lim _{\rho \rightarrow 0} \rho^{1-2 \gamma} \partial_{\rho} U
$$

A second useful lemma is the following (see [CG11], Lemma 4.5 and Theorem 4.7)

Lemma 2.2. There exists a defining function $\rho^{*}$ such that $E\left(\rho^{*}\right)=0$ in the previous lemma. Then we have the following: let $U$ solve

$$
\left\{\begin{aligned}
-\operatorname{div}\left(\left(\rho^{*}\right)^{1-2 \gamma} \nabla U\right) & =0 \quad \\
\left.U\right|_{\rho=0} & =f \quad \text { in }\left(X^{n+1}, g^{*}\right),
\end{aligned}\right.
$$

and derivatives are taken with respect to the metric $g^{*}=\left(\rho^{*}\right)^{2} g^{+}$where we still assume that $\lambda_{1}\left(g_{+}\right) \geq \frac{n^{2}}{2}-\gamma^{2}$. Then,

$$
P_{\gamma}^{\hat{g}} f=-d_{\gamma}^{*} \lim _{\rho^{*} \rightarrow 0}\left(\rho^{*}\right)^{1-2 \gamma} \partial_{\rho^{*}} U+f Q_{\gamma}^{\hat{g}}
$$

Remark 2.1. In the very interesting paper [CC16], the authors establish a link in the extension with conformally covariant operations in metric measured spaces. This generalizes the approach of [CG11].

\section{Short time Existence For General COMPACT MANifolds}

In [JX14], the short time existence of the fractional flow on the sphere follows from an implicit function argument, together with suitable estimates. The argument mainly relies on the fact that the problem is set in $\mathbb{R}^{n}$ (after stereographic projection), and then they can use the representation of the fractional Laplacian as an integral with a singular kernel. In our general case of a compact manifold different from the sphere, such an approach does not seem easy to handle. We follow another route to prove the existence of weak solutions.

The proof of our Theorem 1.1 is done in several steps. In order to prove local wellposedness it is enough to consider the un-rescaled flow, i. e., the following problem

$$
\left\{\begin{array}{l}
\partial_{t} u^{N_{\gamma}}=-P_{\gamma}^{g_{0}} u, \quad \text { for } t>0, x \in M, \\
u(0)=u_{0}
\end{array}\right.
$$


In Lemma 2.1, the zero order term $E(\rho)$ is not suitable for our approach based on a contractivity argument. So instead, we use Lemma 2.2. One then considers the extension to $X$ (the Poincaré-Einsetein manifold) of the function $u$ solving (3.1) denoted $U$ such that then $\left.U\right|_{M}=u$. We drop the harmless constant $d_{\gamma}^{*}$ and rewrite (3.1) as:

Problem (3.1) can be re-written as

$$
\left\{\begin{array}{l}
-\operatorname{div}\left(\left(\rho^{*}\right)^{1-2 \gamma} \nabla U\right)=0 \text { in }\left(X^{n+1}, g^{*}\right), \\
\lim _{\rho^{*} \rightarrow 0}\left(\rho^{*}\right)^{1-2 \gamma} \partial_{\rho} U-U Q_{\gamma}^{g_{0}}=\partial_{t}\left(U^{N_{\gamma}}\right), \text { for } x \in M, t>0 \\
U(0, x)=u_{0}(x), \quad x \in M
\end{array}\right.
$$

Functional spaces. We start by describing the functional spaces needed to define weak solutions. We introduce first the fractional Sobolev space suitable for our purposes. We define the semi-norm

$$
\|f\|_{\dot{H}^{\gamma}(M)}=\int_{M} f P_{\gamma}^{g_{0}} f
$$

Notice that this definition is consistent with the one on the flat case since $P_{\gamma}^{|d x|^{2}}=$ $(-\Delta)^{\gamma}$. In view of the extension by Chang and Gonzalez, we will also need the following weighted Sobolev space

$$
H^{1}\left(M, \rho^{1-2 \gamma}\right)=\left\{f \in L^{2}(M),\left.\left|\rho^{1-2 \gamma}\right| \nabla f\right|^{2} \in L^{1}(M)\right\} .
$$

Notions of WEAK solutions. As already mentioned, we will use the formulation given in (3.2) to handle our problem. We then define a notion of weak solution for this boundary problem, which will serve as a weak formulation for problem (3.1).

Definition 3.1. We say that $u$ in problem (3.1) is a weak solution if its extension $U$ satisfies

(1) $U \in C\left([0, \infty), L^{1}(M)\right), U^{1 / N_{\gamma}} \in L_{l o c}^{2}\left((0, \infty), \dot{H}^{1}\left(M,\left(\rho^{*}\right)^{1-2 \gamma}\right)\right)$

(2) the following identity holds for every test function $\varphi \in C_{0}^{\infty}(\bar{X} \times(0, \infty))$

$$
\int_{0}^{\infty} \int_{M} U^{N_{\gamma}} \partial_{t} \varphi+\int_{0}^{\infty} \int_{M} U Q_{\gamma}^{g_{0}}=\int_{0}^{\infty} \int_{M} \nabla_{g^{*}} U \cdot \nabla_{g^{*}} \varphi
$$

(3) the equality $U(0,)=.u_{0}$ holds a.e.

UNIFORMLY DEGENERATE ELLIPTIC EQUATIONS. An important aspect of our theory relies on understanding the elliptic part of the flows. This is due to our approach based on Time Discretization. This can be done by studying the boundary elliptic problems in Lemma 2.2. We collect here some results and definitions useful for us. We are concerned with the uniformly degenerate elliptic equation

$$
\left\{\begin{aligned}
-\operatorname{div}\left(\left(\rho^{*}\right)^{1-2 \gamma} \nabla U\right)=0 & & \text { in }\left(X^{n+1}, \bar{g}^{*}\right), \\
\left.U\right|_{\rho=0}=f & & \text { on } M^{n} .
\end{aligned}\right.
$$


We use the notations

$$
\begin{aligned}
& B_{R}^{+}=\left\{(x, y) \in \mathbb{R}^{n+1}: y>0,|(x, y)|<R\right\}, \\
& \Gamma_{R}^{0}=\left\{(x, 0) \in \partial \mathbb{R}_{+}^{n+1}:|x|<R\right\}, \text { and } \\
& \Gamma_{R}^{+}=\left\{(x, y) \in \mathbb{R}^{n+1}: y \geq 0,|(x, y)|=R\right\} .
\end{aligned}
$$

In local coordinates on $\Gamma_{R}^{0}$, the metric $\hat{g}$ writes $|d x|^{2}\left(1+O\left(|x|^{2}\right)\right)$ where $x\left(p_{0}\right)=0$. Consider the matrix

$$
A(x, y)=\sqrt{\mid \operatorname{det}\left(\bar{g}^{*}\right)} y^{a}\left(\bar{g}^{*}\right)^{-1}
$$

so that equation (3.3) writes in local coordinates

$$
\sum \partial_{i}\left(A_{i j} \partial_{j} U\right)=0
$$

Furthermore, we have the crucial estimate

$$
A(x, y) \sim y^{a} \alpha(x, y) I d
$$

where $\alpha(x, y)$ is uniformly elliptic.

The weight $y^{a}$ belongs to the Muckenhoupt class $A_{2}$ (see [Muc72]), and the series of papers by Fabes, Kenig, Serapioni and Jerison [FKS82, FJK82] provides a reasonably complete theory for divergence elliptic equations with $A_{2}$ weights. In local coordinates in $\mathbb{R}^{n+1}$, Problem (3.3) with its boundary condition writes

$$
\left\{\begin{array}{cl}
L_{a} U=\operatorname{div}(A \nabla U)=0 & \text { in } B_{R}^{+}, \\
-y^{a} U_{y}=h & \text { on } \Gamma_{R}^{0} .
\end{array}\right.
$$

Definition 3.2. Given $R>0$ and a function $h \in L^{1}\left(\Gamma_{R}^{0}\right)$, we say that $u$ is a weak solution of (3.4) if

$$
y^{a}|A \nabla u \cdot \nabla u|^{2} \in L^{1}\left(B_{R}^{+}\right)
$$

and

$$
\int_{B_{R}^{+}} y^{a} A \nabla u \cdot \nabla \xi-\int_{\Gamma_{R}^{0}} h \xi=0
$$

for all $\xi \in C^{1}\left(\overline{B_{R}^{+}}\right)$such that $\xi \equiv 0$ on $\Gamma_{R}^{+}$.

We have the following results.

Theorem 3.1 (Solvability in Sobolev spaces [FKS82]). Let $\Omega \subset \mathbb{R}^{n+1}$ be a smooth bounded domain, $h=\left(h_{1}, \ldots, h_{n+1}\right)$ satisfy $|h| /|y|^{a} \in L^{2}\left(\Omega,|y|^{a}\right)$, and $g \in H^{1}\left(\Omega,|y|^{a}\right)$. Then, there exists a unique solution $u \in H^{1}\left(\Omega,|y|^{a}\right)$ of $L_{a} u=-\operatorname{div} h$ in $\Omega$ with $u-g \in H_{0}^{1}\left(\Omega,|y|^{a}\right)$.

Theorem 3.2 (Hölder local regularity [FKS82]). Let $\Omega \subset \mathbb{R}^{n+1}$ be a smooth bounded domain and $u$ a solution of $L_{a} u=-\operatorname{div} h$ in $\Omega$, where $|h| /|y|^{a} \in L^{2(n+1)}\left(\Omega,|y|^{a}\right)$. Then, $u$ is Hölder continuous in $\Omega$ with a Hölder exponent depending only on $n$ and $a$. 
Theorem 3.3 (Harnack inequality [FKS82]). Let $u$ be a positive solution of $L_{a} u=0$ in $B_{4 R}\left(x_{0}\right) \subset \mathbb{R}^{n+1}$. Then, $\sup _{B_{R}\left(x_{0}\right)} u \leq C \inf _{B_{R}\left(x_{0}\right)} u$ for some constant $C$ depending only on $n$ and $a$, and in particular, independent of $R$.

Crandall-Liggett Scheme. For our existence proof, we will use the CrandallLiggett idea of implicit discretization in time to reduce the problem of existence of a so-called mild solution for the evolution problem to a cascade of elliptic problems. We will need an existence result plus suitable estimates. We describe the principle of the Crandall-Ligget theorem in our context. Consider equation (3.1),

$$
u_{t}=-P_{\gamma}^{g_{0}}\left(u^{m_{\gamma}}\right)
$$

where we set $u=v^{N_{\gamma}}$, and

$$
m_{\gamma}=\frac{1}{N_{\gamma}}=\frac{N-2 \gamma}{N+2 \gamma}<1
$$

By taking a discrete sequence of times $0<t_{1}<t_{2}<\ldots t_{N}$ and replacing the time derivative by an increment quotient, we reduce the previous evolution problem to a sequence of nonlinear elliptic problems of the iterative form

$$
h P_{\gamma}^{g_{0}} u\left(t_{k}\right)^{m_{\gamma}}+u\left(t_{k}\right)=u\left(t_{k-1}\right), \quad k=1,2, \cdots,
$$

We may take $t_{k}=k h$, and $h>0$ is the time step per iteration. We are led then to study the elliptic problems

$$
h P_{\gamma}^{g_{0}} u^{m_{\gamma}}+u=g
$$

posed in $M$.

ElLIPTIC ANALYSis. Next, we perform the analysis of this elliptic problem. We consider only the case $Q_{\gamma}^{g_{0}} \geq 0$. There is no lack of generality in putting $h=1$ in the previous argument.

Theorem 3.4. Let $g \in L^{1}(M) \cap L^{\infty}(M)$. Then there exists a unique weak solution $U \in H_{l o c}^{1}\left(X,\left(\rho^{*}\right)^{1-2 \gamma}\right)$ to

$$
\left\{\begin{array}{cl}
\operatorname{div}\left(\left(\rho^{*}\right)^{1-2 \gamma} \nabla U\right)=0 & \text { in } X \\
-\lim _{\rho^{*} \rightarrow 0}\left(\rho^{*}\right)^{1-2 \gamma} \partial_{\rho^{*}} U+Q_{\gamma}^{g_{0}} U+U^{N_{\gamma}}=g & \text { on } M
\end{array}\right.
$$

Furthermore, if $U$ and $\tilde{U}$ are two weak solutions, one has the inequality

$$
\int_{M} Q_{\gamma}^{g_{0}}(U-\tilde{U})_{+}+\int_{M}\left(U^{N_{\gamma}}-\tilde{U}^{N_{\gamma}}\right)_{+} \leq \int_{M}(g-\tilde{g})_{+} .
$$

Proof. Pick a point $p \in \partial_{\infty} X$ and consider the problem in local coordinates around $p$ in a geodesic half-ball $B_{R}^{+} \subset X$

$$
\left\{\begin{array}{c}
\operatorname{div}(A(x, y) \nabla U)=0 \quad \text { in } B_{R}^{+} \\
-\lim _{y \rightarrow 0} y^{1-2 \gamma} \partial_{y} U+Q_{\gamma}^{g_{0}} U+U^{N_{\gamma}}=g
\end{array} \text { on } \Gamma_{R}^{0}\right.
$$


We add homogeneous Dirichlet boundary conditions on $\Gamma_{R}^{+}$. A weak solution $U$ of (3.10) satisfies

$$
\int_{B_{R}^{+}} A(x, y) \nabla U \cdot \nabla \varphi+\int_{\Gamma_{R}^{0}} Q_{\gamma}^{g_{0}} U \varphi+\int_{\Gamma_{R}^{0}} U^{N_{\gamma}} \varphi=\int_{\Gamma_{R}^{0}} g \varphi,
$$

for each $\varphi$ compactly supported. A way to produce a weak solution is to minimize

$$
J(U)=\frac{1}{2} \int_{B_{R}^{+}} A(x, y) \nabla U \cdot \nabla U+\frac{1}{2} \int_{\Gamma_{R}^{0}} Q_{\gamma}^{g 0} U^{2}+\frac{1}{N_{\gamma}+1} \int_{\Gamma_{R}^{0}} U^{N_{\gamma}+1}-\int_{\Gamma_{R}^{0}} U g .
$$

The functional is coercive by the Poincaré inequality (see [FKS82] for the Poincaré inequality for $A_{2}$ weights), the Sobolev trace embedding (see [FKS82]) and CauchySchwarz inequality. Recall here that the weight $A(x, y)$ is $A_{2}$ in $X$.

We now establish the contractivity. Let $U$ and $\tilde{U}$ be two solutions with data $g$ and $\tilde{g}$. Consider in the weak formulation the test function $\varphi=p(U-\tilde{U})$ where $p$ is any smooth monotone approximation of the sign function, $0 \leq p(s) \leq 1$ and $p^{\prime}(s) \geq 0$. Then we have by testing the weak formulation

$$
\begin{gathered}
\int_{\Omega} p^{\prime}(U-\tilde{U}) A(x, y) \nabla(U-\tilde{U}) \cdot \nabla(U-\tilde{U})+\int_{M} Q_{\gamma}^{g_{0}}(U-\tilde{U}) p(U-\tilde{U}) \\
+\int_{M}\left(U^{N_{\gamma}}-\tilde{U}^{N_{\gamma}}\right) p(U-\tilde{U})=\int_{M}(g-\tilde{g}) p(U-\tilde{U}) .
\end{gathered}
$$

Using the fact that

$$
A(x, y) \geq C y^{1-2 \gamma}>0 .
$$

and passing to the limit in the test function as $p$ tends to the Heaviside function $\widehat{p}=\operatorname{sign}^{+}$, we get the desired inequality. This is what Bénilan calls $T$-contractivity, Ben72] and it implies both contractivity and the comparison principle.

The proof of the existence theorem then follows from the fact mild solutions are weak solutions as in dPQRV12.

Remark 3.1 (Extinction in finite time of the un-rescaled flow). The previous unrescaled flow extinguishes in finite time. The proof is contained in dPQRV12 in the Euclidean setting but carries out to the manifold case. The only point to check is the existence of a Stroock-Varopoulos inequality, i.e. let $\gamma \in(0,1)$ and $q>1$ then

$$
\int_{M}\left(|v|^{q-2} v\right) P_{\gamma / 2}^{g} v \geq\left.\frac{4(q-1)}{q^{2}} \int_{M}\left|P_{\gamma / 4}^{g}\right| v^{q / 2}\right|^{2}
$$

The Stroock-Varopoulos inequality is a general inequality on Dirichlet spaces. Our operator $P_{\gamma}^{g}$ generates a Dirichlet form in $L^{2}(M)$ (see [Bak94]). 
3.0.1. Proof of Theorem 1.1. The proof is as follows: given $u_{0}$, we compute a solution $u(x, \tau)$ of the un-rescaled flow (3.1). The rescaled flow satisfies

$$
\left\{\begin{array}{l}
\partial_{t} v^{N_{\gamma}}=-P_{\gamma}^{g_{0}} v+a(t) v^{N_{\gamma}} \quad t>0, x \in M \\
v(0)=v_{0}
\end{array}\right.
$$

and the flow is volume preserving. Now, if $F$ denotes

$$
\frac{d F}{d t}=a(t)
$$

then it is easy to see that the solution $u(x, \tau)$ satisfies

$$
\left\{\begin{array}{l}
\partial_{\tau} u^{N_{\gamma}}=-P_{\gamma}^{g_{0}} u \quad t>0, x \in M \\
u(0)=u_{0},
\end{array}\right.
$$

provided

$$
\frac{d \tau}{d t}=e^{F(t)\left(1-\frac{1}{N_{\gamma}}\right)}
$$

and furthermore we have $\int_{M} u(x, \tau) e^{F(t)}=$ constant, giving

$$
e^{F(\tau)}=\frac{\text { const }}{\int_{M} u(t, \tau)} .
$$

Hence, from $u(x, \tau)$, we compute $\int_{M} u(t, \tau)$, which gives $F(\tau)$, giving $v(x, t)$ by the relation

$$
v=e^{F} u,
$$

hence the solution to the rescaled flow.

\section{Global existence And proof of Theorem 1.2}

Let $F$ be the stereographic projection from $\mathbb{S}^{n}$ into $\mathbb{R}^{n}$ with north pole $q_{0} \in \phi(V)$,i.e. the inverse of

$$
F^{-1}(x)=\left(\frac{2 x}{1+|x|^{2}}, \frac{|x|^{2}-1}{|x|^{2}+1}\right), x \in \mathbb{R}^{n}
$$

and we set $q_{0}=(0, \cdots, 1)$. Define $w$ as

$$
\left(F^{-1}\right)^{*} \tilde{g}=w^{4 / n-2 \gamma} g_{\mathbb{R}^{n}} .
$$

Then the function $w$ satisfies the equation

$$
\left\{\begin{array}{l}
\partial_{t} w^{N_{\gamma}}=-(-\Delta)^{\gamma} w t>0, x \in \mathbb{R}^{n} \\
w(0)=w_{0}
\end{array}\right.
$$

Note that the function $w_{0}$ is only defined on $\mathbb{R}^{n} \backslash F(\Gamma)$ and

$$
\lim _{x \rightarrow F(\Gamma)} w_{0}(x)=+\infty .
$$

This equation has been investigated in dPQRV12. We can assume that the manifold $M$ is not conformally covered by the sphere. Indeed this case has been investigated in JX14. 
By a deep theorem by Schoen and Yau SY88, since we assume that the locally conformally flat manifold has positive Yamabe constant, there exists a conformal diffeomorphism $\phi$ from the universal cover $\tilde{M}$ of $\left(M,\left[g_{0}\right]\right)$ onto a dense domain $\Omega$ of the sphere $\mathbb{S}^{n}$. Thus the manifold $\left(M,\left[g_{0}\right]\right)$ is the quotient of $\Omega$ under a Kleinian group and $\Gamma=\partial \Omega$ is the limit set of this group (note that $\Gamma \neq \emptyset$ ). We set

$$
\tilde{g}=\left(\phi^{-1}\right)^{*} \pi^{*} g
$$

and

$$
\tilde{g}=\tilde{u}^{\frac{4}{n-2 \gamma}} g_{\mathbb{S}^{n}}
$$

where $\pi: \tilde{M} \rightarrow M$ is the covering map and $g_{\mathbb{S}^{n}}$ is the round metric on the sphere. Therefore, by construction, the metric $\tilde{g}$ solves (1.2) and by conformality, $\tilde{u}$ solves (1.7) with $g_{0}$ replaced by $g_{\mathbb{S}^{n}}$.

The following lemma is a corollary of Proposition 2.6 in [SY88].

Lemma 4.1. Let $M$ be locally conformally flat manifold with non-negative Yamabe invariant. Assume equation (1.7) has a local solution defined on $(0, T)$ for some $T>0$. Then for any $\tilde{t} \in[0, T)$, we have, uniformly in $t \in[0, \tilde{t}]$ :

$$
\lim _{x \rightarrow \Gamma} \tilde{u}(x, t)=+\infty .
$$

Given $p_{0} \in M$, we choose a point $\tilde{p}_{0} \in \tilde{M}$ and a neighborhood $V$ of $\tilde{p}_{0}$ such that $\pi\left(\tilde{p}_{0}\right)=p_{0}$ and $\operatorname{dist}(\phi(V), \Gamma)>0$. Then there is some $C>0$ such that

$$
\tilde{u}_{0}=\tilde{u}(., 0)>C^{-1}
$$

and $\tilde{u}_{0}$ is smooth on $\phi(V)$.

The final step to prove Theorem 1.2 is the following theorem.

Theorem 4.2. Let $M$ be locally conformally flat manifold with non-negative Yamabe invariant. Let $u$ be a solution (1.7). Then for any $t \geq 0$, one has the Harnack inequality

$$
\sup _{x \in M} u \leq C \inf _{x \in M} u \text {. }
$$

The proof of Theorem 4.2 follows the approach by Ye Ye94. As a consequence of the volume-preserving character of the flow, there exists $\alpha, \beta>0$ such that for any $t \geq 0$ one has for any $x \in M$

$$
\alpha<u(t, x)<\beta .
$$

Hence the solution exists globally and the theorem is proved, is smooth by the results in $\mathrm{VdPQR}$ and the convergence of the flow is ensured by the well-known results of Simon Sim83. We now come to the proof of Theorem 4.2,

This is based on the following theorem

Theorem 4.3. Let $\gamma \in(0,1)$ and assume that $M$ is locally conformally flat with nonnegative fractional curvature. Then there exists $C$ not depending on $u$ such that

$$
\sup _{M} \frac{\left|\nabla_{g_{0}} u\right|}{u} \leq C \text {. }
$$


Proof. The proof follows the argument of YYe94. The solution $\tilde{u}(t, x)$ has the expansion at infinity

$$
\tilde{u}(t, x)=\frac{2^{(n-2 \gamma) / 2}}{|x|^{n-2 \gamma}}\left(a_{0}+\frac{a_{i} x_{i}}{|x|^{2}}+\left(a_{i j}-\frac{n-2 \gamma}{2} \delta_{i j}\right) \frac{x_{i} x_{j}}{|x|^{4}}+O\left(|x|^{-3}\right)\right) .
$$

A similar expansion holds for the derivatives of $\tilde{u}$ :

$$
\begin{gathered}
\frac{\partial \tilde{u}}{\partial x_{i}}(t, x)=2^{(n-2 \gamma) / 2}\left(-\frac{n-2 \gamma}{|x|^{n-2 \gamma+2}} x_{i}\left(a_{0}+\frac{a_{j} x_{j}}{|x|^{2}}\right)+\frac{a_{i}}{|x|^{n-2 \gamma+2}}-\frac{2 x_{i} a_{j} x_{j}}{|x|^{n-2 \gamma+4}}\right. \\
\left.+O\left(|x|^{-(n-2 \gamma+3)}\right)\right)
\end{gathered}
$$

where

$$
\begin{gathered}
a_{0}(s)=u\left(q_{0}, t\right), \\
a_{i}(s)=\frac{\partial(u \circ G)}{\partial x_{i}}(0)
\end{gathered}
$$

and

$$
a_{i j}(s)=\frac{\partial^{2}(u \circ G)}{2 \partial x_{i} x_{j}}(0)
$$

where $q_{0}$ denotes the north pole and $G$ the inverse of the stereographic projection.

Denoting $y_{i}(t)$,

$$
y_{i}(t)=\frac{a_{i}}{(n-2 \gamma) a_{0}}
$$

and $y(s)=\left(y_{1}(s), \ldots, y_{n}(s)\right)$, it is enough to prove a uniform bound on $y$ in the local existence range. Fix a time $T$. After a rotation and a reflection, we may assume

$$
y_{n}(T)=\max _{i}\left|y_{i}(T)\right| \text {. }
$$

A standard argument gives that for some $\lambda_{0}>0$ we have: for each $\lambda>\lambda_{0}$ the following holds

$$
\tilde{u}(x, 0)>\tilde{u}\left(x^{\lambda}, 0\right) x_{n}<\lambda
$$

where $x^{\lambda}=\left(x_{1}, \ldots, x_{n-1}, 2 \lambda-x_{n}\right)$, the reflection point w.r.t the hyperplane $\left\{x_{n}=\lambda\right\}$. Note that here we have a singular set $F(\Gamma)$ for $\tilde{u}(x, 0)$. However, thanks to Lemma 4.1, the previous estimate holds.

We may assume that $F(\Gamma)$ lies strictly below the plane $\left\{x_{n}=\lambda_{0}\right\}$. We now follow the proof of [JX14]. By proposition 2.3 in [JX14], one has uniformly in $[0, T)$

$$
\tilde{u}(x, t)>\tilde{u}\left(x^{\lambda}, t\right) \quad x_{n}<\lambda, \lambda \geq \lambda_{0} .
$$

We claim that

$$
\max _{s \in[0, T]} y_{n}(s)<\lambda_{0}
$$

If not, there exists $\tilde{s} \in(0, T]$ such that $y_{n}(\tilde{s})=\max _{0 \leq s \leq T} y_{n}(s) \leq \lambda_{0}$. Setting $\lambda=y_{n}(\tilde{s})$ one gets after defining $\bar{u}(x, s)=\tilde{u}\left(x+y_{n}(\tilde{s}), s\right)$

$$
\bar{u}\left(x^{\prime}, x_{n}, s\right)>\bar{u}\left(x^{\prime},-x_{n}, s\right) s \in[0, T], x_{n}<0 .
$$


Then taking Kelvin transforms $\bar{u}_{1}(x, s)=\frac{1}{|x|^{n-2 \gamma}} \bar{u}\left(x /|x|^{2}, s\right)$ (satisfying the same equation) we have

$$
\bar{u}_{1}\left(x^{\prime}, x_{n}, s\right)>\bar{u}_{1}\left(x^{\prime},-x_{n}, s\right) s \in[0, T], x_{n}<0 .
$$

Invoking proposition 2.8 in [JX14, one has

$$
\frac{\partial\left(\bar{u}_{1}\left(x^{\prime}, x_{n}, s\right)-\bar{u}_{1}\left(x^{\prime},-x_{n}, s\right)\right.}{\partial x_{n}}<0 \quad(x=0, s=\tilde{s}) .
$$

This contradicts the asymptotic expansion of $\tilde{u}(x+y, s)$. Hence to derive the Harnack inequality, the previous argument gives

$$
\frac{\left|\nabla_{\mathbb{S}^{n}} \tilde{u}\right|}{\tilde{u}} \leq C
$$

on $\Phi\left(V^{\prime}\right)$ where $V^{\prime}$ is a neighborhood of $p_{0}$ with $V^{\prime} \subset \subset V$. Now, by Lemma 4.1, this leads

$$
\frac{\left|\nabla_{g_{0}} \tilde{u}\right|}{\tilde{u}} \leq C
$$

on $\pi\left(V^{\prime}\right)$. Since $M$ is compact, one can cover $M$ by finitely many $V^{\prime}$ and up to enlarging $C$ this gives the theorem.

\section{ACKNOWLEDGMENTS}

P. Daskalopoulos has been partially supported by NSF grant DMS-1600658 and J.L. Vázquez by Project MTM2014-52240-P (Spain). The second author would like to acknowledge the hospitality of the department of Mathematics of Columbia University where part of this work was discussed.

\section{REFERENCES}

[Bak94] Dominique Bakry. L'hypercontractivité et son utilisation en théorie des semigroupes. In Lectures on probability theory (Saint-Flour, 1992), volume 1581 of Lecture Notes in Math., pages 1-114. Springer, Berlin, 1994.

[Ben72] P. Benilan. Equations d'évolution dans un espace de banach quelconque et applications. Ph. D. Thesis, Univ. Orsay (in French), 1972.

[Bre02] Simon Brendle. A generalization of the Yamabe flow for manifolds with boundary. Asian J. Math., 6(4):625-644, 2002.

[Bre05] Simon Brendle. Convergence of the Yamabe flow for arbitrary initial energy. J. Differential Geom., 69(2):217-278, 2005.

[Bre07] Simon Brendle. Convergence of the Yamabe flow in dimension 6 and higher. Invent. Math., 170(3):541-576, 2007.

[CC16] Jeffrey S. Case and Sun-Yung Alice Chang. On fractional GJMS operators. Comm. Pure Appl. Math., 69(6):1017-1061, 2016.

[CG11] Sun-Yung Alice Chang and María del Mar González. Fractional Laplacian in conformal geometry. Adv. Math., 226(2):1410-1432, 2011.

[Cho92] Bennett Chow. The Yamabe flow on locally conformally flat manifolds with positive Ricci curvature. Comm. Pure Appl. Math., 45(8):1003-1014, 1992.

[CL71] M. G. Crandall and T. M. Liggett. Generation of semi-groups of nonlinear transformations on general Banach spaces. Amer. J. Math., 93:265-298, 1971. 
[CLO06] Wenxiong Chen, Congming Li, and Biao Ou. Classification of solutions for an integral equation. Comm. Pure Appl. Math., 59(3):330-343, 2006.

[CS07] Luis Caffarelli and Luis Silvestre. An extension problem related to the fractional Laplacian. Comm. Partial Differential Equations, 32(7-9):1245-1260, 2007.

[dPQRV11] Arturo de Pablo, Fernando Quirós, Ana Rodríguez, and Juan Luis Vázquez. A fractional porous medium equation. Adv. Math., 226(2):1378-1409, 2011.

[dPQRV12] Arturo de Pablo, Fernando Quirós, Ana Rodríguez, and Juan Luis Vázquez. A general fractional porous medium equation. Comm. Pure Appl. Math., 65(9):1242-1284, 2012.

[Esc92] José F. Escobar. Conformal deformation of a Riemannian metric to a scalar flat metric with constant mean curvature on the boundary. Ann. of Math. (2), 136(1):1-50, 1992.

[FG02] Charles Fefferman and C. Robin Graham. Q-curvature and Poincaré metrics. Math. Res. Lett., 9(2-3):139-151, 2002.

[FJK82] E. Fabes, D. Jerison, and C. Kenig. The Wiener test for degenerate elliptic equations. Ann. Inst. Fourier (Grenoble), 32(3):vi, 151-182, 1982.

[FKS82] E. B. Fabes, C. E. Kenig, and R. P. Serapioni. The local regularity of solutions of degenerate elliptic equations. Comm. Partial Differential Equations, 7(1):77-116, 1982.

[GJMS92] C. Robin Graham, Ralph Jenne, Lionel J. Mason, and George A. J. Sparling. Conformally invariant powers of the Laplacian. I. Existence. J. London Math. Soc. (2), 46(3):557-565, 1992.

[GMS12] Maria del Mar González, Rafe Mazzeo, and Yannick Sire. Singular solutions of fractional order conformal Laplacians. J. Geom. Anal., 22(3):845-863, 2012.

[GQ13] María del Mar González and Jie Qing. Fractional conformal Laplacians and fractional Yamabe problems. Anal. PDE, 6(7):1535-1576, 2013.

[GZ03] C. Robin Graham and Maciej Zworski. Scattering matrix in conformal geometry. Invent. Math., 152(1):89-118, 2003.

[Ham88] Richard S. Hamilton. The Ricci flow on surfaces. In Mathematics and general relativity (Santa Cruz, CA, 1986), volume 71 of Contemp. Math., pages 237-262. Amer. Math. Soc., Providence, RI, 1988.

[JX14] Tianling Jin and Jingang Xiong. A fractional Yamabe flow and some applications. $J$. Reine Angew. Math., 696:187-223, 2014.

[KMW] S. Kim, M. Musso, and J. Wei. Existence theorems of the fractional yamabe problem. Preprint, https://arxiv.org/abs/1603.0661\%.

[Li04] Yan Yan Li. Remark on some conformally invariant integral equations: the method of moving spheres. J. Eur. Math. Soc. (JEMS), 6(2):153-180, 2004.

[Muc72] B. Muckenhoupt. Weighted norm inequalities for the Hardy maximal function. Trans. Amer. Math. Soc., 165:207-226, 1972.

[QR06] Jie Qing and David Raske. Compactness for conformal metrics with constant $Q$ curvature on locally conformally flat manifolds. Calc. Var. Partial Differential Equations, 26(3):343-356, 2006.

[Sim83] Leon Simon. Asymptotics for a class of nonlinear evolution equations, with applications to geometric problems. Ann. of Math. (2), 118(3):525-571, 1983.

[SS03] Hartmut Schwetlick and Michael Struwe. Convergence of the Yamabe flow for "large" energies. J. Reine Angew. Math., 562:59-100, 2003.

[SY88] R. Schoen and S.-T. Yau. Conformally flat manifolds, Kleinian groups and scalar curvature. Invent. Math., 92(1):47-71, 1988.

[VdPQR] Juan-Luis Vázquez, Arturo de Pablo, Fernando Quiros, and Ana Rodriguez. Classical solutions and higher order regularity for nonlinear fractional diffusion equations. To appear in JEMS.

[Ye94] Rugang Ye. Global existence and convergence of Yamabe flow. J. Differential Geom., 39(1):35-50, 1994. 
P. Daskalopoulos, Department of Mathematics Columbia University 2990 Broadway New York, NY 10027

pdaskalo@math.columbia.edu

Y. Sire, Department of Mathematics Johns Hopkins University 3400 N. Charles Street

Baltimore, MD 21218 sire@math.jhu.edu

J. L. Vázquez, Departamento de Matemáticas, Universidad Autónoma de Madrid, Campus de Cantoblanco, 28049 Madrid, Spain juanluis.vazquez@uam.es 